Mara Viveros Vigoya

Universidad Nacional de Colombia

\title{
Políticas de sexualidad juvenil y diferencias étnico-raciales en Colombia: reflexiones a partir de un estudio de caso
}

\begin{abstract}
Resumen: Este artículo analiza las modalidades de 'gobierno de la sexualidad juvenil' puestas en obra por medio de las a ctividades educativas realizadas en los programas de salud sexual y reproductiva destinados a jóvenescolombia nos, y sus efec tos en la producc ión de subjetividades juveniles. Exa mina ta mbién las percepciones de quienes rea lizan estas intervenciones so bre las diferencias étnic o-racia les existentes entre jóvenes y su incidencia en el quehacer de las y los func iona rios implic a dos en estos programas. La información se basa en la observación de dos experiencias de intervención en salud sexual y reproductiva c on jóvenes esc ola riza dos en Bogotá, y en las entrevistas a los y los coordina dores de los talleres de educ a ción sexual y a las personas encargadas de las labores de consejería en cada uno de los programas.
\end{abstract}

Palabrasclave: sexualidad juvenil, salud sexual y reproductiva, derechos sexuales, diferencias etnic o-raciales, Colombia.

Copyright $\odot 2006$ by Revista Estudos Feministas

${ }^{1}$ FASSIN y MEMMI, 2004.

2 FOUCAULT, 1988.

\section{Introducción}

La intervención social en relación con la salud sexual y reproductiva de los jóvenes en Colombia expresa lo que Didier Fassin y Dominique Memmi ${ }^{1}$ llaman el gobiemo de los cuerpos, es decir, la intromisión de los poderes públicos en la relación privada del individuo con su destino físic o a tra vés de códigos, regla mentos, normas, valores, relaciones de autoridad y de legitimidad. Estos a utores retoman la palabra 'gobiemo' en el amplio sentido en que la usó Michel Foucault ${ }^{2}$ para designar las formas de acción orienta dasa actuar sobre las posibilidades de acción de los otros. En este caso, el gobierno de la sexualidad juvenil haría referencia a la forma en que se dirige y estructura el posible campo de acción de los y las jóvenes en materia sexual. 


\begin{abstract}
${ }^{3}$ La asociación Pro-Bienestar de la familia colombiana, Profamilia, una entidad privada, a filiada a la Federación Intemacional de Planific a ción Familiar (IPPF), brinda el $65 \%$ de la Planificación familiar en el país a través de treinta y cinco centros (Marcela Sánchez, 2003). En 1990 creó e primer centro para jóvenes en la ciudad de Bogotá en 1990 buscando responder a la necesidades específic as de los (as) jóvenes en materia de sexualidad.
\end{abstract}

El documento presentado se basa en la observación de dos experiencias de intervención en salud sexual y reproductiva con jóvenes escolarizados en Bogotá, realizadas por dos entidades, una pública y otra privada, el Programa de educación en Sexualidad Humana de la Universidad Nacional de Colombia, Sede Bogotá, y el Programa de jóvenes de Profa milia, sede Kennedy. ${ }^{3}$ En cada uno de estos programas se rea lizó la observación de cinco ta lleres impartidos a jóvenes estudia ntes de secunda ria y a 'primíparos' universitarios en el área de promoción y prevención en Salud sexual y reproductiva, se efectuaron entrevistas a los (as) coordinadores (as), a los responsables de los talleres y a las personas encargadas de las labores de consejería en sus respectivas instituciones (diez en total).

Si prefiero ubicar esta reflexión sobre la gestión de la sexualidad de las y los jóvenes en un marco referido al gobiemo de los cuerpos y no al de la salud es porque considero, siguiendo a estos autores, que este tema desborda su acotación en el ámbito de la salud y las práctic as profesionales de los médic os e incluye las formas en que distintos agentes e instituciones sociales (los profesionales paramédicos, los funciona rios que ejecutan las polític as públicas, los educadores, la fa milia, la Ig lesia, el Estado, los medios de comunic ación etc.) definen, perciben y regulan las representaciones y los comportamientos relacionados con la sexualidad de los y las jóvenes.

Abordar la intervención sobre la sexualidad juvenil desde este punto de vista permite ir más allá de los recortes administra tivos de la salud, e incluir un a mp lio espectro de a rbitrajes públic os potenc iales en este ámbito. Hace posible además, dar cuenta de las transformaciones contemporáneas de las biopolíticas, entendidas como los modos de racionalizar, ya sea en téminos de conocimientos o de acciones, los problemas que plantean a la sociedad los jóvenes, constituidos como grupo poblacional, en relación con su fecundidad, morbilidad y mortalidad por causas sexuales o reproductivas. Pero también, examinar el biopoder que se ejerce sobre los jóvenes a través de estas intervenciones, no sólo desde sus objetivos normalizadores, sino desde su incidencia en sus procesos de subjetivación, es decir en las formas en que se transforman a sí mismos (as) en sujetos.

Este trabajo busca aportar a la reflexión sobre las actividades educativas realizadas en los programas de salud sexual y reproductiva destinados a las y los jóvenes colombianos. En él se ofrece un análisis sobre las modalidades particulares de gobierno de los cuerpos puesto en obra por estas intervenciones y sobre sus efectos 


\footnotetext{
${ }^{5}$ Un ejemplo de este tipo lo brinda el recurso al reclutamiento de multiplic adores para los talleres de educación sexual entre los mismos jóvenes, buscando que los asistentes al taller se sientan identific ados con quienes lo coordinan.
}

en la producción de subjetividades juveniles. Igualmente, se indican las percepciones de quienes realizan estas intervenciones sobre las diferencias étnico-raciales existentes entre jóvenes y su incidencia en el quehacer de las y los funcionarios implic ados en estos programas.

\section{Modalidades actuales del gobiemo de la sexualidad juvenil}

La historia de la salud pública muestra múltiples tentativas de norma lizar las prácticas sociales, entre ellas la sexualidad, y de poner en marcha el proyecto de gobierno de los cuerpos a prehendidos en forma individual o colectiva. Estas tentativas han adoptado modalidades muy variadas que van desde los intentos de control muy directos hasta la utilización de dispositivos orientados a persua dir a ciertas poblaciones de adecuar sus formas de vida según criterios saludables, pasando por numerosas situa c iones intermedias. ${ }^{4}$

En tiempos recientes, la adolescencia y la juventud han suscitado una creciente atención de parte de las orga nizaciones de salud internacionales y de gobiemos y organizaciones no gubernamentales, nacionales, regionales y locales, por ser considerado un grupo poblacional particularmente expuesto a desarrollar comportamientos de riesgo, nefastos para su salud. Estos comportamientos pueden incluir prácticas sexuales sin protección que pueden llevar a una maternidad o patemidad tempranas o a infecciones o enfermeda des de transmisión sexual - en algunos casos mortales como el vih-sida - y comprometer su presente y su futuro y el desarrollo de la sociedad. A nivel de la acción, se han definido estrategias para promover la salud de las y los jóvenes que parten del supuesto de que ésta depende cada vez más de su conducta y de la inclusión de grupos claves de su entomo social que influyen en ellas. ${ }^{5}$

Las formas de vigilancia que se despliegan a ctualmente en torno a las conducta s sexuales de los y las jóvenes se construyen con base en la transferencia que se les hace de las decisiones relativas a la administración y protección de su salud, el control de su reproducción, la construcción de su proyecto de vida, la escogencia de su estilo de vida, etc. Así lo expresan las líneas de acción en la promoción de la salud sexual y reproductiva de los y las adolescentes, incluidas en la política nacional de salud sexual y reproductiva para el período 2002-2006. En estas líneas de acción se le pide al "adolescente" - como una categoría universal indiferenciada - "que lleve a cabo acciones de autocuidado centradas en la doble 
${ }^{6}$ FASSIN y MEMMI, 2004.

${ }^{7}$ FOUC AULT, 1991, p. 35.

${ }^{8}$ Michel BOZON, 2002; y Christian AUTHIER, 2002.

${ }^{9}$ BERLIVET, 2004.

${ }^{10}$ BERLVET, 2004, p. 44.

${ }^{11}$ BOZON, 2002. protección"; "que se apropie de la posibilidad de retardar la edad de inicio de las rela ciones genitales, la frecuencia con que éstas se tiene, y la edad de la concepción". Estas nuevas formas de gobiemo de los cuerpos buscan que cada uno de ellos se cuide y se vigile lo mejor posible, y sancionan a aquél que se muestra incapaz de hacerbuen uso de esta delegación de poder o tiene actitudes y comportamientos 'desviados' que perjudican el acuerdo social establecido en tomo de la norma. ${ }^{6}$ Es importante señalar que aunque en la práctica la solicitud de autocuidado y la evaluación de ciertos comportamientos y a c titudes juveniles como desvia dos es diferente en función de criterios de género, los funciona rios que aplican dichas políticas no se han apropiado de una perspectiva de género en el ejercicio de sus funciones.

La autovigilancia que realizan los y lasjóvenespara adaptarse a las definiciones de la normalidad, transformánd ose y modificándose en ese proceso, se hace mediante un amplio espectro de tecnologías del yo, definidas como "aquellas técnicas que permiten a los ind ividuos efectuaruna serie de operaciones en sus propios cuerpos, en sus almas, en sus pensamientos, en sus conductas... con el fin de alcanzar un cierto estado de perfección..."7 En lasúltimas tres o cuatro déca das del siglo XX a sistimos a cambios en la sexua lidad que corresponden más a una dinámica de individualización de los comportamientos y losideales que a una revolución sexual, en el sentido de una transformación radical del orden sexual. ${ }^{8}$

La educación que se busca impartir ha dejado de serpercibida como una intervención externa sobre el sujeto para garantizar el cumplimiento de la norma y a pensarse en términos de una serie de acciones públicas orientadas hacia una mejor administración de la salud sexual de las poblacionesjóvenes, a tra vés de la neutraliza ción de ciertos comportamientos considera dos funestospara su sa lud. ${ }^{9}$ Este segundo régimen de intervención política es el de los 'dispositivos de seguridad' que Michel Foucault distingue de los mecanismos disciplinarios. Mientras éstos últimos actúan 'desde el exterior' para imponer la norma hasta en los pliegues más recónditos de los comportamientos individuales, los dispositivos de seguridad "no pueden instituirse sino a condic ión de hacer jugar 'desde el interior' ciertos aspectos del fenómeno, considerado de forma tal que se neutra licen sus efectos más deletéreos". ${ }^{10}$ Según la tesis de la individua lización de la sexualidad, estos últimos decenios han acelerado el proceso de sustitución de controles y disciplinas externas por controles y disciplinas internas que acrecientan las exigencias sociales). ${ }^{11} \mathrm{El}$ 
12 FOUCAULT, 1999, p. 247.

${ }^{13}$ J osé Femando SERRANO, 2004.

${ }^{14}$ FOUCAULT, 1988 y 1999. gobiemo de las conductas sexuales juveniles también se entiende mejor si se considera la crítica foucaldiana al núcleo del poder concebido como regla, prohibición, ley y como lo que marca el límite entre lo permitido y lo no permitido.

Si bien la mayoría de las reflexiones sobre el poder se han realizado a partir de una concepción jurídica del mismo que intenta explicar dónde está el poder, quién lo detenta, qué reglas lo administran, etc, lo que Foucault muestra con maestría son las muta ciones tec nológ ic as del poder en Occidente. Actualmente, este poder se ejerce, ya sea a través de tecnologías individualizantes, la a natomopolítica, o de tecnologías que no apuntan a los individuos sino a las poblaciones, la biopolítica. El gobiemo de la sexualidad de los jóvenes requiere tanto de las disc ip lina sind ividua les del cuerpo y su acción correccional como de las regulaciones colectivas de su sexualidad, como forma de normalizar la reproducción de las poblaciones. En este cruce de caminos, entre la anatomopolítica y la biopolítica, el gobierno de la sexua lidad de los jóvenes constituye "una pieza política de primera magnitud para hacer de la sociedad una máquina de producción"12 y para orientar el curso vital de los jóvenes hacia una vida adulta modelada por la disciplina moral y familiar. ${ }^{13}$

\section{Técnicas y procedimientos para instaurar este gobiemo}

Una de las técnicas de poder para instaurar este gobiemo es la que Foucault llama "poder pastoral", que en sus modalidades modemas deja de pretender guiar a la gente para su sa Iva ción en el más allá, para a segurarsu salva ción en este mundo, 'haciendo el bien' en el sentido más material del término, es decir, procurando la salud, el bienestar, la seguridad, entre otras. ${ }^{14}$ Algunas de las características de este tipo de poder que se pueden encontrar en las instituciones que se ocupan de la salud pública sexual y rep roductiva de las y los jóvenes son, entre otras, las siguientes: la autorida d para obligara cada joven a hacer lo necesario para mantenerse saludable; la preocupación por asegurar no sólo el bienestar de cada uno y cada una de ellos como individuo sino como grupo poblacional; la movilización de diferentes a c tores (médic os, psicólogos, trabajadores sociales, educadores), para llevar a delante estas funciones pastorales. Por último, la puesta en marcha de mecanismos de poder y control sobre la sexualidad, presentada, como la perpetua fuente, en el interior de los individuos, de situaciones de riesgo para su 
15 En Colombia se creó el Plan Nacional de Educación Sexual, liderado por el Ministerio de Educación desde el año de 1993. Su evaluación, rea liza da en 1998 señala la persistencia de temores y resistencia de parte de maestros y padresen relación con su abordaje de forma directa y explícita y "la consecuente capacitación deficiente de quienes tienen la responsabilidad de su implementa ción" (SDSB - Secreta ría Distrita de Salud de Bogotá, 2004, p. 27) Igualmente, destaca el énfa sis de la información en los riesgos de ejercicio sexual, principalmente los relacionadoscon los embarazosprecocesy las enfermedades de transmisión sexual (ETS). salud, aprehendida en consonancia con la moral dominante.

El ejercicio de este poder pastoral se hace aún más necesario en el caso de los y las jóvenes si consideramos que el retroceso del control de los padres sobre los hijos en este momento del curso de vida transfiere en la práctica la regulación de la sexualidad juvenil a losparesy a losmedios de comunic ación, principales fuentes de informa ción para los jóvenes sobre cuestiones ligadas a la sexualidad. Las enseñanzas informa les que esta s fuentes imparten han sido percibidas a menudo por los adultos como insuficientes, erróneas e incluso mal orientadas, razón por la cual se ha buscado desarrolla r proyec tos de educación sexual ${ }^{15}$ que complementen o se opongan a ciertas informaciones y actitudes, y que busquen modificaciones en las representaciones y en los comportamientos sexuales de los jóvenes desfavorables para su salud.

En Colombia, país en el que la Iglesia católica ha ejercido una fuerte influencia, la educación sexual ha sido un proyecto difícilmente aceptado y ha tenido numerosos obstáculos de diverso orden para su desarrollo, por considerarse que incita a los jóvenes al ejercicio sexual indisc rimina do. Entidades como PROFAMILIA, institución pionera en la atención de la salud sexual y reproductiva, y el Servic io de Salud de la Universidad Nacional de Colombia han continuado esta labor educativa como parte sus programas de atención de la salud sexual y reproductiva de las y los a dolescentes y jóvenes. Una de las moda lida des escogidas para desa rrolla r sus estra teg ias de ca pa cita ción, información y educación ha sido la de los talleres de educación sexual, que analiza ré a continuación.

El programa de sexualidad humana de la división de salud de la Universidad Nacional de Colombia, sede Bogotá, está conformado por un equipo integrado pordos enfermeras, una médica y tres psicólogas. Como parte de sus acciones de información, orientación y atención en salud sexual efectúa cada semestre, durante la llamada semana de inducción, talleres titula dos "reflexiones sobre la sexualidad", con los estudiantes del primer semestre académico de los distintos programas de cada Facultad. El objetivo de estos encuentros es "propiciar el a nálisis de actitudes y valores inherentes al fomento de criterios de autocuidado que contribuyan a la vivencia de una sexualidad sana, responsable y gratificante".

Los talleres se inician a partir de un planteamiento general sobre la sexualidad, como un asunto complejo multifa cétic o y transversa I al curso de vida. Sin embargo, a medida que se desarrollan los contenidos del taller, las referencias a la sexualidad se concentran en un período 
${ }^{16}$ Kennedy es una de las veinte localidades en que se divide administra tivamente el distrito capital de Bogotá. Es una de las localidades con mayor porcentaje de población joven, un $62 \%$ de la misma no supera los treinta años, y en edad escolar. específico del ciclo de vida, "la edad reproductiva", y en las relaciones que pueden tener como consecuencia embarazos precoces, enfermedades e infecciones de transmisión sexual. El final del tallercirc unscribe aún más el tipo de información brindada, enfatizando los aspectos técnicos de los riesgos que trae el ejercicio sexual, en rela ción con los emba razos 'tempra nos' y las enfermeda des e infecciones de transmisión sexual.

Los contenidos educativos se acompañan de una serie de imágenes de las lesiones provocadas por estas enfermedades e infecciones que suscitan generalmente reacciones de preocupación y rechazo. En síntesis, la informa ción de carác ter téc nic 0 , y el impacto que generan la simá genes terminan por encubrir e inhibir el examen y la discusión de la informa ción presentada y de la visión de la sexualidad ofrecida en el taller.

El forma to de los ta lleres rea liza dos por Profa milia en los colegios de la localidad de Kennedy'16 se adapta a un público adolescente, cua tro o 5 años más joven que el de la Universidad, adecuando las estrategias pedagógicas, el tono y lenguaje y os contenidos mismos del taller. Los talleres buscan resolver los problemas planteados por la insuficiente $\mathrm{y} / \mathrm{o}$ errada información que generalmente tienen las y los adolescentes en asuntos sexuales y reproductivos e incentivar la expresión libre de sus dudas en estas materias. Sin embargo, por ser realizados en los mismos locales escolares, durante la jomada educativa y con la presencia a veces inoportuna de sus docentes, los talleresno logran romper las ba rerasque impone el entomo escolar a la formulación de este tipo de inquietudes.

Por otra parte, es necesario precisar que los talleres en los colegios no sólo buscan cumplir una función educa tiva sino eliminar "los obstá c ulos so cio-culturales" que dificultan el acceso de las y los a dolescentes a la a tención en salud sexual y reproductiva. Por tal razón los coordina dores de los talleres vinculan consta ntemente las preocupaciones que tímidamente formulan las y los alumnos a las respuestas que ofrece Profamilia como institución prestadora de servicios de salud. Dicho de otra manera, los talleres constituyen el primer paso hacia la consulta médica impartida en el Centro para jóvenes de la localidad.

Aunque los talleres aluden repetidamente a los distintos a spectos involucrados en la sexualidad, su perspectiva es predominante psicológica y médica. La primera se manifiesta en las descripciones de la actividad sexual como una manifestación de la vida psíquica regida por leyes propias y en la orienta ción de los talleres hacia el fortalecimiento de habilidades psicosociales para la 
elección de métodos de anticoncepción y protección, y de actitudes activas hacia el autocuidado. La segunda perspectiva se expresa en la redefinición de las prácticas y comportamientos sexuales en términos médicos cuyo corolario es convertirlos en procesos clínicos susceptibles de exigir exámenes, diagnósticos y tra ta mientos médicos y susc itar polític as de sa lud públic a. ${ }^{17}$

Estas dos perspectivas, a unque se presentan como técnic as, es decir como neutra saxiológ ic a mente, proponen una aproximación individualizante y normativa a la sexualidad juvenil al buscar generar en cada joven la capacidad de evaluar sus comporta mientos sexuales con razonamientos prescriptivos, así sean los de ejercer una sexua lidad "libre y sa tisfactoria" pero "responsable y sana". Por otra parte se articulan muy bien con el modelo existente de salud privatizada, sometida a la racionalidad del mercado, a sus esquemas de análisis y a sus criterios de decisión y donde las mayores responsabilidades son delegadas a los individuos. Predomina entonces una perspectiva psic ologizada, medic a lizada, y privatiza da que deja muy poco espacio a la sexualidad como un ámbito de la vida social susceptible de ser pensado porla teoría social o como un terreno político, de construcción de ciudadanía y de movilización social.

Las a c ciones educ a tiva s rea liza das en estos ta lleres producen un tipo partic ula rde subjetividad juvenil. No tanto la de la joven y el joven ciudadanos, sujetos de derechos sexuales y reproductivos particulares sino la de las y los jóvenes a decua dos a las normas colectivas en ma teria de sexualidad y usuarios potenciales de los servicios de salud. Además de transferiruna informa ción, los talleres de a mbas entidades pretenden reforzar la conciencia de sí de las y los jóvenes; propiciar en ellas y ellos un discurso a sertivo, informado y maduro sobre la sexualidad, otorgando importancia al tema de la prevención de riesgos. Se les instiga en consecuencia a afirmar su individualidad en ruptura con los comportamientos miméticos que pueden serperjudicia lespara la salud y lasideas nocivas difund idas por los medios de comunicación y el entomo inmediato.

Las actitudes de las coordinadoras de los talleres están en consonancia con los objetivos consigna dos en el acápite de la promoción de la salud sexual y reproductiva de los adolescentes de la Política nacional de salud sexual y reproductiva: lograr que "se cuestionen los modelos sociales y culturales que posicionan el embarazo precoz como una forma de ganarestatus o reconocimiento social o a fec tivo" y que "se apropien de la posibilidad de retardar la edad del inicio de las relaciones genitales, la frecuencia con que éstas se tienen y la edad de la concepción" ${ }^{18}$ En 
19 BERLVET, 2004.

20 Claudio STERN y Gabriel MEDINA, 1999; y Eleonor FAUR, 2003a y 2003b, entre otros.

${ }^{21}$ Phillipe ARIÉS, 1973; Norbert ELÍAS, 1998; Martine SEGALEN 1981; Rodrigo PARRA SANDOVAL 1985; Luz Gabriela ARANG O 1991 y 1992; J osé Femando SERRANO 1998; y Sonia MUÑOZ, 1999, entre otros.

\footnotetext{
${ }^{22}$ Carles FEIXA, 1998, p. 18.
}

resumen, el proceso educativo en los talleres le deja poco espacio a la sexualidad como una experiencia positiva para los jóvenes y generadora de placer. Pese al enunciado de la importancia de ejercer una sexualidad en forma libre y satisfactoria, se sigue haciendo un mayor énfasis en su control que en su conocimiento o disfrute.

En los talleres flota siempre la idea de que el sexo es a lgo inadecuado e inoportuno en este momento del curso vital, una a ctividad sospechosa que requiere ser contenida y vigilada. Los talleres se desa rrollan en tomo de una ideaclave, aparentemente contradictoria, que proclama que a uto-limitarse sexualmente es para las y los jóvenes ganar un espacio de libertad. ${ }^{19}$ De este modo, la postergación del inicio de las relaciones sexuales como forma de prevención de los embarazos, las ETS y las infecciones de transmisión sexual (ITS) se presenta como una afirmación de la independencia frente a las presiones de los pares y del entorno social. Sin embargo, este aparente signo de libertad no es sino una expresión de sujeción, ya no como sometimiento a un otro por el control y la dependencia sino como constitución de una subjetividad a partir de la sumisión a unas normas interiorizadas que se superponen con la a utoconciencia.

\section{Las realidades múltiples de la sexualidad adolescente y juvenil y la salud sexual y reproductiva}

La concepción de adolescencia y juventud que manejan los programas de salud sexual y reproductiva son determinantes en las orientaciones de los programas desarrollados por las instituciones prestadoras de servicios de salud. Numerosos tra ba jo ${ }^{20}$ han seña la do las dific ulta des de una definición de adolescencia y juventud, basada en criterios psicológic os y biológ ic os que a porta $n$ a rgumentos difusos y precarios para establecer el inic io y el término de esta fase del ciclo vital. Desde una perspectiva histórica y sociológic ${ }^{21}$ se ha planteado que esta noción, estrechamente asociada a la expansión de la educación secundaria, designa una nueva etapa de la vida que prepara para la adultez y pospone el ejercicio de las funciones laborales, proc rea tivasy parenta les que le están a socia das. Las a proxima ciones a ntropológ ic as indic a n por su parte que la juventud aparece como una construcción cultural en el tiempo y en el espacio y que aunque cada sociedad organiza la transición de la infancia a la vida adulta, la forma y contenido de esta transición son extremadamente variables. ${ }^{22}$ 
${ }^{23}$ Mara VIVEROS, 2003.

${ }^{24}$ Inés Elvira MEJ ÍA MOTTA, 2000.

${ }^{25}$ SERRANO, 2004.
En el caso de las investiga ciones sobre la sexualidad juvenil, es importante señalar además que una gran parte de ellas se han efectuado desde un enfoque de salud y quien dice salud, habla de normaliza ción. En este sentido, el deseo y los placeres sexuales juveniles han sido concebidos como los de unos sujetos incompletos, en vías de formación, y porlo tanto no aptos para respondera los requerimientos de una sexualidad responsable y plena como se supone es la sexualidad adulta. ${ }^{23}$ Igualmente, los tra bajos señalan a los jóvenes como una población de alto riesgo para la prevención en salud sexual y reproductiva, por un inicio de su vida sexual con un bajo nivel de información, sin ningún tipo de protección y en un contexto social marcado porcategoría s como el pecado, la culpa, el machismo y la subordinación de la mujer. ${ }^{24} \mathrm{C}$ on base en estos diagnósticos, la investigación reciente sobre jóvenes ha sido realizada desde una perspectiva que subraya el ajuste o desajuste de sus formas de sery a ctuar en rela ción con las normas del mundo adulto, utilizado como patrón de referencia para calific ar lo juvenil. Este "a dulto centrismo" ha bría determina do y legitima do a demás las polític a s los programas socia les orienta dos hacia los jóvenes. ${ }^{25}$ Por otra parte, cuando se incluye en estas investigaciones e intervenciones sociales el tema del cuerpo de los jóvenes, éste se reduce muchas veces a los órganos genitales y reproductivos, y se ve como un cuerpo biológic o y no como un cuerpo a través del cual se incorpora el sí mismo que caracteriza a la persona, se expresa la relación con el mundo y se viven las regulaciones, vigilancias y controles efec tua dos por las distinta instituc iones so c ia les (la fa milia, la escuela, la Iglesia, entre otras).

La juventud, como un sectorsocial delimita do a partir de la edad es una cuestión que ha sido fuertemente debatida pormuchos de los a utores a nteriormente cita dos. Si bien algunos de ellos señalan que la edad es un dato que sólo cobra sentido histórica y culturalmente, ta mbién admiten que es un referente empírico inevitable en la biog ra fía de los individuosy en el a nálisis de los signific a dos que se le a tribuyen No obstante, es prec iso considerar que la edad es una variable demográfica que no define una especificidad particular de los sujetos ya que estos se construyen en el complejo entramado de las relaciones socia les de clase, género, pertenencia étnico-racial, local y cultural, donde la edad no es sino uno de los múltiples factores que entran en juego.

A continuación se muestra cómo perciben los funcionarios que coordinan y desarrollan los programas a nalizados, una de las estructuras socia les que diferencian a los jóvenes que participan en ellos, la etnia/"raza", y de 
qué forma incide en el quehacer profesional de los funciona rios.

\section{La perspectiva étnico-racial en la educación sexual en el contexto del multic ultura lismo}

En contraste con la categoría de género, incorporada por mandato institucional en los dos programas, las diferencia sétnico-ra ciales entre los jóvenes son poco percibidas y a sumid as por sus funcionarios, pese a la existencia de un nuevo marco normativo e interpreta tivo, c rea do a partir de la a firma ción constituc ional de la plurietnicidad y la multiculturalidad de la nación colombiana en 1991. La adopción de la perspectiva de género en las políticas y los programas de salud sexual y reproductiva ha tenido que ver más con la incorporación de un mandato institucional, fruto de las presiones de las agencias financiadoras externas que con un compromiso construido a partir de las necesidades sentidas por las instituciones o con un nuevo enfoque para analizar y abordar los eventos sexuales y reproductivos.

En términos generales, podríamos decir que las y los funcionarios manifestaron cierta resistencia a nuestra pregunta sobre la pertinencia de incluir una dimensión étnico-racial en la formulación de sus programas de educación sexual y reproductiva, por considerar que diferenciar a lasy los jóvenes porca teg oría sétnico-raciales era equivalente a introducir un criterio discriminatorio en los programas. Incluso, alguno de ellos señaló que esas diferencias dan lugara "prebendas injustific a dasya que a veces, los miembros de los grupos étnicos no son los más necesitados de apoyo porparte de las instituciones" y que la precariedad está más asociada con las condiciones de clase que con una pertenencia étnico-racial. Otros respondieron a nuestra pregunta en forma bastante defensiva: "Aquí a todosse atienden porigual", "la salud es un derecho que tienen todos los jóvenes por igual".

Las y los funciona rios entrevistados a sumieron con gran prevención cua lquier alusión a lo racial, y de manera a ntic ipa da se protegieron de cualquiera cusación personal

${ }^{26}$ Franklin GIL, 2004. 0 institucional de racismo. ${ }^{26}$ Esta actitud defensiva debe leerse también como una denegación del racismo, práctica bastante común en Colombia, donde se ejerce cotid ia namente un ra cismo sutil, de baja intensidad, trivial y tan incorporado en las relaciones interpersonales, que se ha vuelto casi imperceptible. Muchos ciudadanos colombianostoman la actitud de no reconocerla existencia del racismo en esta sociedad, porque éste se asocia a 
27 VIVEROS, 2004.

${ }^{28}$ Eduardo RESTREPO, 2004. una agresión violenta, instituciona liza da o espontánea de un grupo racial hacia otro y no a una práctica de tipo individual que adopta una forma más de exclusión que de agresión. ${ }^{27}$

No obstante, en la medida en que íbamos a dentrándonos en las descripciones de los programas, las y los funciona rios entrevista dos caían en la cuenta de que en a mba instituc iones se a tendían jóvenes indígena sy a frocolombianos: en la Universidad existe un programa de admisión especial para estudiantes procedentes de municipios pobres en el cual partic ipan muchos estudiantes indígenas y en la localidad de Kennedy, en la cual está ubicado el programa de Profamilia analizado, reside un porcentaje relativamente importante de población afrocolombiana. Las referencias a estos dos grupos de jóvenes se hacen más en términos de grupos étnicos, con rasgos culturales propios, asociados muchas veces a subculculturas regionales, que en términos "raciales", o sociales. Llama la atención también que se suele hacer hincapié en las diferencias culturales existentes entre jóvenes pero no así en las similitudes ni en las condiciones de vivienda, salud y otros aspectos cotidianos precarios, comunes a muchos de ellos y ellas.

Desde una representación 'lineal' del desarrollo, las supuestas diferencias culturales son construidas como rasgos tra dic iona les y pre-modemos, y como signos de unas concepciones "étnicas", a tá vicas y retrógradas, en contraste con las normas sexuales y de género vigentes en Bogotá, una ciudad presentada como "occidental", urbana, modema y no marcada "étnicamente". En estas dic otomías, tra dic ional/modemo, étnico/no étnico, el primer término es ubicado en un campo semántico subordinado, mientras el segund o se convierte en el punto de referencia, en el lugar social no marca do y por lo tanto naturalizado. Por otra parte, es importante considerar la forma en que ciertos gestos, deseos y cuerpos son 'etnizados' bajo relaciones de poder específicas y el modo en que la etnicidad constituye "normalidades" y "a normalidades" sociales. ${ }^{28}$ Desde esta perspectiva deben analizarse las descripciones de una funcionaria de la Universidad Nacional que define a los estudiantes indíg enas como más tímid os y reserva dos que los otros y con "referentes fa milia res más fuertes en sus discursos"; igualmente, como más distantes del servicio de salud y más renuentes al uso del preservativo en sus relaciones sexuales. 0 sus referencias "al machismo y a la promiscuidad prevaleciente en las comunidades a fro-colombia nas", a simila da smuc has veces a las poblaciones provenientes de las costas. 
${ }^{29}$ Donny MEERTENS, 2002, p. 18.

${ }^{30} \mathrm{GIL}, 2004$, p. 17
Conviene seña lar que en los últimos a ños y frente al fenómeno creciente del desplazamiento forzado de sus regiones de origen por situaciones de violencia, de numerosas poblaciones, los llamados "desplazados" son el grupo objetivo privilegiado de los programas de salud sexual y reproductiva, principalmente en lo que se refiere a la promoción de métodos anticonceptivos definitivos. Es importante precisar además que en el conjunto de las y los desplazados existe una sobre-representación de población desplazada afro-colombiana e indígena. En el caso de Bogotá podemos señalar de manera indicativa que entre la población que acudió a la Unidad de Atención Integral a Población Desplazada del Distrito, cerca del 9\% era afro-colombiana. ${ }^{29}$

En relación con la perspectiva étnico-racial, es difícil identific ar si la orienta ción de las a cciones en salud sexual y reproductiva desarrolladas con la población desplazada hacia el suministro de métodos definitivos de antic onc epción está determinada "por las representaciones estereotipadas sobre las 'proles numerosas' de las poblaciones rurales, por el deseo de minimizar la precariedad de sus condiciones económic as, poruna nueva forma de estigmatización social a sociada al desplazamiento o por una combinación compleja de todas las razones anteriores". ${ }^{30}$ En realidad, en este tipo de decisiones, se cruzan inextricablemente consideraciones acerca de la clase y el origen regional, en el que por razones históricas se encubren en Colombia, las diferencias étnico-raciales.

El ámbito en el que más se ha incorporado la temátic a de las diferencias étnic o-racia les existentes entre las y los jóvenes colombianos es el del mercadeo publicitario de los programas de salud sexual y reproduc tiva. Recientemente se ha desarrollado una campaña publicitaria que busca promover que las mujeres carguen el condón, tratando de cuestionar una serie de prejuicios morales frente a las mujeres que lo cargan, por denotar explícitamente que son sexualmente activas. Como lo refiere la persona encargada de liderar esta estrategia publicitaria: "el objetivo de la campaña es que todas las mujeres, de todos los colores, de todas las razas, de todas lasformas, digan que el condón están dispuestas a carga rlo ellas". Las dos primeras imágenes de esta campaña han estado representadas por una mujer negra joven, peinada a la manera "rasta", pero ambientada en un contexto urbano y modemo, y la segunda, por una conocida joven ac triz 'blanca', prota gonista de una serie televisiva centrada en las y los jóvenes de sectores populares.

Estas estrategias han integrado muy bien la caracterización del país como una nación pluriétnica y 
${ }^{31}$ Charles TAYLOR, 1994.

${ }^{32}$ Nancy FRASER, 1997.

33 HARDT y NEGRI, 2002, citados en VIVEROS, 2004.

${ }^{34}$ Paul RABINOW, 1984. multicultural. En efecto, esta caracterización ofrece un nuevo lenguaje, el de la etnicidad, que permite resignific ar las diferencias y convertirlas en a tributos culturales valorizados ${ }^{31}$ desde una perspectiva celebratoria de la diversidad y generar expresiones gráfic as representa tivas de la pluralidad de la población colombiana como algo valioso. El problema de esta perspectiva es que elogia la diferencia sin preguntarse por su relación con la desigualdad, como si todos los grupos fueran socialmente iguales y como si la diferencia perteneciera únicamente a la cultura. ${ }^{32}$ En el caso colombiano, es necesario recordar además que las reivindicaciones identitarias de los movimientos indígenas y afrocolombianos no han estado desligadas de una aspiración al ejercicio de una ciudadanía común.

El 'respeto' a la diversidad cultural está a plicándose en el ámbito public ita rio como consecuencia y adecuación de las estra tegias y contenidos de la comunicación a los mandatos del marketing contemporáneo y a la dinámica corporativa en el mercado mundial. En estos tiempos de globa liza ción, las estra tegias public ita rias 'p rog resistas' inc luyen las 'minorías' raciales, étnic as, polític as, religiosas, de género, de orienta ción sexual, etc., en laspropagandas y en las poblaciones objetivo de su mercadeo con el fin de lograr una mejor 'administración de la diversidad' y una organiza ción de sus 'energías diferenciadas' en aras de la ganancia empresarial. ${ }^{33}$

\section{Reflexiones finales}

El gobiemo de la sexualidad juvenil apela, como he señalado en el texto, a 'dispositivos de seguridad' que busc an neutra liza r los comp orta mientos sexua les juveniles percibidos como nefastos para su salud. Estos dispositivos permiten el ejercicio de un tipo particular poder, el que llama Foucault el poderpastoral, ejercido por instituciones que buscan procurar el bienestar y la salud simultá nea mente en los individuos y los grupos poblacionales, y a cuden a tec nologías que pueden ser consideradas como tec nologías de norma liza ción. ${ }^{34}$

El ejercicio de este poder pastoral garantiza igualmente la producción y constitución de unos sujetos que se auto-controlan y auto-disciplinan para adaptarse a las definiciones de la normalidad, transformándose y modificándose en ese proceso, mediante un amplio espectro de tecnologías del yo. Este proceso de subjetivación se hace siguiendo un modelo de juventud que define el curso vital de manera gradual y sucesiva, y vuelve prisioneros a los sujetos de unas esc a las tempora les 
35 LESKO, 2001, citado en SERRANO, 2004, p. 47.

${ }^{36}$ FAUR, 2003a.

${ }^{37}$ Maria Betania ÁVILA, 1999 determinadas. Este modelo se orienta con base en el patrón escolar que crea correspondencias entre determinadas edades y ciertos momentos del desa rrollo psicológico y moral, y determina las temporalidades adecuadas para cada evento sexual y reproductivo.

Una de estas tecnologías de normalización de los cuerpos de los y las jóvenes es la de los talleres de educación sexual en los que prevalece como se indicó en a parta dos a nteriores una naturaliza ción del sexo como un objeto pre-social y previo a la cultura, una idea de cuerpo como da to biológico, un modelo dicotómico de los sexos y una percepción de la condición juvenil como una situación disociada de la historia y la cultura y "de las condiciones de clase que determinan a quiénes se aplica o no tal condición juvenil". ${ }^{35}$

El gobiemo de la sexualidad juvenil tiene un carácter pa radójico: poruna parte, en teoría transfiere las decisiones referidasa la administra ción y protección de la salud sexual y reproductiva a los propios jóvenes. Pero, por otra, mantiene las relaciones de dependencia a socia das al lugar que ocupan las y los jóvenes en los sistemas productivos, reproductores de la subordinación de lo juvenil al mundo adulto. También se perciben tensiones entre un discurso que busca promoversu capacidad reflexiva y susactitudes responsables y activas hacia el autocuidado y una participación débil de las y los a dolescentes y jóvenes en la afirmación y defensa de sus derechos sexuales y reproductivos. El fortalecimiento de esta participación requiere tanto de espacios propicios para la escucha e incorporación de sus voces como de la capacidad de ellas y ellos como sujetos que puedan dar cuenta de esa voz propia. ${ }^{36}$

Aunque la reflexión sobre la ciudadanía y los derechos ya está en el escenario de la salud sexual y reproductiva aún sigue siendo un componente débil. Si bien en la política nacional de salud sexual y reproductiva se habla de fortalecer la participación de los jóvenes tanto individual como colectivamente, en la práctica sabemos que la mayoría de los jóvenes tiene muy poca ciudadanía y menos en materia sexual y reproductiva: Las y los jóvenes no son pensados desde el Estado como sujetos políticos que definen sus proyectos y tienen la posibilidad de participary actuar en la esfera polític a en la cual se toman las dec isiones sobre los intereses colec tivos. ${ }^{37}$ No tienen voz ni voto en la formulación de políticas y programas que supuesta mente están diseña dospara beneficiarlos. Cuando participan en la educación de sus congéneres, como en el caso de algunos programas de Profamilia, lo hacen en calidad de multiplicadores, para hacer más a trayentes los 
${ }^{38}$ Bonnie SHEPARD, 2004, p. 143.

talleres, pero sin estar dotados de los instrumentos necesarios para incidir en los enfoques y contenid os de las acciones de información y educación en materia sexual. Su partic ipa ción en los prog ra ma ses simbólic a y dec ora tiva, pero no está contribuyendo a construir ciudadanía.

En los últimosaños, a la educa ción sexual que acude a losmedios de comunic a ción public itaria (folletos, a fic hes, videos, cuñas radiales y televisivas), se le ha asignado un lugar importante como una forma de acción pública que puede llegara un amplio público y contribuir a transformar actitudes y comportamientos sociales. Sin embargo, es importante diferenciar la potencialidad de las imágenes audiovisuales para extender el alcance de la difusión de campañas sobre prevención de la salud reproductiva de su poderpara fomentarmayorparticipación e interacciones en los y las jóvenes a propósito de estas campañas. No obstante, los procesos comunica cionales ma sivos pueden ser un recurso para hacer presentes los intereses de las y los jóvenes y propiciar la defensa de sus derechos sexuales y reproductivos.

La influencia de la iglesia católica en el estado colombiano, pese a la abolición del concordato y al reconocimiento constitucional de la pluralidad religiosa, sigue siendo preponderante. Y en el caso de las políticas públicas relacionadas con la salud sexual y reproductiva ha sido nefasta, oponiéndose a la provisión masiva de preservativos para prevenir la infección del VIH-SIDA y a la disponibilidad de la anticoncepción de emergencia por considerarla una técnica abortiva; promoviendo la abstinencia (denegando la realidad de la actividad sexual de losjóvenes); estableciendo equivalencias entre servicios preventivos y promoción de inmoralidad y promiscuidad. Estas normas culturales y religiosas conservadoras que se oponen a la amplia y universal disponibilidad de información y servicios para jóvenes se constituyen en obstáculos para el ejercicio de esta ciudadanía. ${ }^{38}$

Las entidades prestadoras de servicios de salud sexual y reproductiva han recibido de las agencias internacionales que las fina ncian el manda to de incorporar una perspectiva de género en sus políticas y programas. Aunque esta perspectiva tendría como objetivo proponer un nuevo enfoque para analizar y abordar los eventos sexuales y reproductivos, en la práctica no ha contribuido a transformar signific a tivamente las relaciones sociales de sexo ni a promover la equidad de género en las políticas y programas de salud reproductiva. El tratamiento de las diferencias de género ha sido más en términos de a dministra rlas que de brindar herra mientas para responder a las necesidades diferenciales de las y los jóvenes. Si en 
${ }^{39}$ RESTREPO, 2004, p. 92

${ }^{40}$ FRASER, 1997. este á mbito no se han hecho log ros, aún menosa delanta do está el proceso de visibilización de los efectos que tiene el ordena miento jerárquico de las diferencias étnico-raciales existentes entre las y los jóvenes sobre su salud sexual y reproductiva. Tampoco existe, en la s entid a des presta doras de servicios de salud sexual y reproductiva, conciencia de la utilización de estas diferencias para naturalizar y reproducir las desigualdad es sociales en materia de salud sexual y reproductiva. Es importante tener en cuenta que "en la producción de los cuerpos dóciles, las diferencias somáticas y de comportamiento entre los individuos son producidas, registradas, finamente medidas, distribuidas y fijadas de acuerdo con la norma". ${ }^{39}$

El fortalecimiento de una ciudadanía en la adolescencia y juventud requiere desarrollar algunas estrategias particula res que den cuenta de la multiplicidad de rea lidadesque se oc ultan detrás de la categoría 'joven', cuando se define únicamente desde la dimensión etárea. Existe, no obstante, una tensión entre la protección de derechos que responda a las particularidades de cada grupo, y el riesgo de construir lecturas de estas diferencias en términos esencialistas. Esta tensión subraya la importancia de construir ma rcos norma tivos que contengan simultáneamente políticas de redistribución - para luchar contra las desigualda des soc ioec onómic a s existentes entre los distintos grupos de jóvenes - y políticas de reconocimiento - para fomentar el respeto de sus diferencias, como lo señala Nancy Fraser al sustentar su concepción de una democracia radical. ${ }^{40}$ Se trata entonces de desplegar políticas y programas de salud sexual y reproductiva que fortalezcan la voz y la capacidad de decisión de todos y cada uno de las y los jóvenes, deconstruyendo los patrones de valoración desigual en términos de sus múltiples diferencias. Se trata también de proponer prácticas educativas que dejen de imponer normas universales de comportamiento sexual y criterios prescriptivos de su organización. Se trata de generar las condic iones para que las y los jóvenes puedan construirse como sujetos de sus conductas morales y hacer uso de los plac eresal servic io de su estiliza ción y no de su codific ación.

\section{Referencias bibliográficas}

ARANGO, Luz Gabriela. "Socialización, adolescencia e identidad de género en sectores populares urbanos". Proyecto Colcultura-Icetex. Programa de Becas Francisco de Paula Santander. Bogotá, mayo 1991. Informe final. 
"Esta tus adolescente y valores a sociados con la matemida d y la sexualid a d". In: FASSIN, Did ier; DEFOSSEZ, Anne-Cla ire; VIVEROS, Mara (Eds.). Mujeres de los Andes. Condiciones de vida y salud. Bogotá: IFEA/Universidad Externado de Colombia, 1992. p. 263-287.

ARIÉS, Philippe. L'enfant et la vie familiale sous I'Ancien Régime. Paris: Seuil, 1973.

AUTHIER, Christian. Le nouvel ordre sexuel. Paris: Bartillat, 2002.

ÁVILA, Maria Betania. "Feminismo y ciudadanía: la producción de nuevos derechos". In: SCAVONE, Lucila (Comp.). Género y salud reproductiva en América Latina. Cartago, Costa Rica: LUR, 1999. p. 57-83.

BERLVET, Luc. "Une biopolitique de l'éducation pour la sa nté. La fabrique des campagnes de prévention". In: FASSIN, Didier; MEMMI, Dominique (Dirs.). Le gouvernement des corps. Paris: Editions de l'Ecole des Hautes Etudes en Sciences Sociales, 2004. p. 37-75.

BOZON, Michel. Sociologie de la sexualité. Paris: Nathan, 2002.

ELAS, Norbert. La civiliza ción de los padres y otros ensayos. Bogotá: Ed itorial Norma, 1998.

FASSIN, Did ier; MEMMI, Dominique. "Le gouvemement de la vie, mode d'emploi". In : FASSIN, Didier; MEMMI, Dominique (Dirs.). Le gouvernement des corps. Paris: Editions de l'École des Hautes Etudes en Sciences Sociales, 2004. p. 9-33.

FAUR, Eleonor. "Derechos y Ciudadanía. Proyec to Adolescencia en América Latina y el Caribe. Orientaciones para la formulación de políticas". In: OLAVARRIA, José (ed.). Varones adolescentes: género, identida des y sexua lida des en Améric a La tina. Santia go: FNUAP/FLAC SO, 2003a. p. 315-327.

"¿Escrito en el cuerpo? Género y derechos humanos en la adolescencia". In: CHECA, Susana (Comp.). Género, sexualidad y derechos reproductivos en la a dolescencia. Buenos Aires: Paidós, 2003b. p. 37-76.

FEIXA, Carles. El reloj de arena. Estudio de Culturas juveniles. México: Dirección General Causa Joven-Centro de Investiga ciones y Estudios so bre la J uventud, 1998. n. 4.

FOUC AULT, Michel. "El sujeto y el poder". In: RABINOW, Paul; DREYFUS, Hubert (Eds.). Más allá del estruc turalismo y la hermenéutica. Méxic 0: UNAM, 1988. p. 239 et seq.

Estética, ética y hermenéutica. Buenos Aires/ Barcelona: Editorial Paidós, 1999. Obras Esenciales, v. III.

FRASER, Na nc y. lustic ia Interrupta. Reflexiones c rític as desde la posición post-socialista. Bogotá: Siglo del Hombre/ Uniandes, 1997. 
GIL, Franklin. Insumos para la elaboración del informe final. Proyecto de investigación "Evaluación cua litativa de programas de SSR en poblaciones jóvenes de Bog otá y Cali (una mirada de género, clase, 'color de piel' y orientación sexual)". Bogotá: Universidad Nacional de Colombia. Centro de Estudios Sociales. Escuela de Estudios de Género, julio 2004.

MEERTENS, Donny. Encrucijadas urbanas: población desplazada en Bogotá y Soachas, una mirada diferenciada por género, edad y etnia. Bogotá: Alto Comisiona do de Naciones Unidas para los Refugiados (Acnur), 2002.

MEJ ÍA MOTTA, Inés Elvira. Dinámicas, ritmos y significa dos de la sexualidad juvenil. Bogotá: Programa La Casa/ CESO Universidad de los Andes, 2000.

MUÑOZ, Sonia. Jóvenes en disc usión. Sobre edades, rutinas y gustos en Cali. Bogotá: Funda ción Social/Fundación Restrepo Barco/Procívica TV/Fundación FES, 1999.

PARRA SANDOVAL, Rodrigo. Ausencia de futuro. La juventud colombiana. Bogotá: CEPAL/ Plaza y Ja nés, 1985.

RABINOW, Paul. The Foucault Reader. New York: Panteón, 1984.

RESTREPO, Eduardo. Te orías contemporáneas de la etnicidad. Stuart Hall y Michel Foucault. Popayán: Editorial Universidad del Cauca, 2004.

SÁNCHEZ, Marcela. “Derechos y Ciudadanía. Proyec to Servicios para Adolescentes. Posibilidades para el ejercicio de sus derec hos sexua les y reproductivos". In: OLAVARIA, José (ed.). Varones adolescentes: género, id entida des y sexualida des en América La tina. Santia go: FNUAP/FLACSO, 2003. p. 327-333.

SDSB - SECRETARÍA DISTRITAL DE SALUD DE BOGOTÁ D.C. Modelo de servicios de atención integral en salud sexual y reproductiva para adolescentes y jóvenes del Distrito Capital. Investigadora principal: Lucía Castro Cabrera. Bogotá D.C, marzo de 2004.

SEGALEN, Martine. Sociologie de la famille. Pa ris : Armand Colin, 1981.

SERRANO, José Femando. "La investigación sobre jóvenes: estudios de (y desde) las culturas". In: MARTíN, Jesús; LÓPEZ, Fa bio (Eds.). C ultura, medios y sociedad. Bogotá: Ces/Universidad Nacional, 1998. p. 274-309.

. Menos querer más de la vida. Concepciones de vida y muerte en jóvenes urbanos. Bogotá: Siglo del Hombre Editores, Universidad Central-DIUC, 2004.

SHEPARD, Bonnie. "Pecado y derechos humanos. La falta de ciudadanía sexual de la juventud". In: CÁCERES, Carlos; FRASCA, Tina; PECHENY, Mario; TERTO, Veria no (Eds.). Ciudadanía sexual en América Latina. Abriendo 
el debate. Lima: Universidad Peruana Cayeta no Heredia, 2004. p. 141-153.

STERN, Claudio; MEDINA, Gabriel. "Adolescencia y salud en Méxic o". In: OLIVEIRA, Maria Coleta (Org.). Cultura, adolescencia, saúde. São Paulo: Consorcio La tinoamericano de Programas em Saúde Reprodutiva e Sexualidade, 1999. p. 98-160.

TAYLOR, Charles. Multiculturalismo. Différence et démocratie. Paris: Aubier, 1994.

VIVEROS, Mara. "Orientaciones íntimas en las primeras experiencias sexuales y amorosas de los jóvenes. Reflexiones a partir de algunos de estudios de casos colombianos". In: OLAVARRÍA, José (Ed). Varones adolescentes: género, identidades y sexualidades en Améric a Latina. Santia go: FNUAP/FLACSO, 2003. p. 115127.

VIVEROS, Mara. "Nuevas formas de representación y viejos estereotipos raciales en los comercia les public ita rios colombianos". In: VIVEROS, Mara; LAVOU ZOUNGBO, Victorien (Eds). Mots pour Nègres. Maux de Noir(e)s. Enjeux socio-symboliques de la nomination des Noir(e)s en Amérique Latine. Perpignan: CRILAUP/Presses Universita ires de Persignan, 2004. p. 79-101.

[Rec ebido em agosto de 2005 e aceito para publicação em abril de 2006]

\section{Adolescent Sexuality Policy and Ethnic/Racial Differences in Colombia}

Abstract: This artic le a nalyzes the particular modes of governance of a dolescent sexua lity that emerge from the educ ational ac tivities carried out by sexual and reproduc tive health programs for young Colombians. It a Iso a nalyzes their effects on the production of a dolesc ent subjec tivities. In addition, it examines the perception of those who carry out the pedagog ic ac tivities related to sexuality with adolescents and the way these perceptions are marked by ethnic/racial differences, as well as their influence on their professional performance. Field da ta a re based on observation of two pilot programs on sexual and reproduc tive health among adolesc ent high sc hool students in Bogotá, and on interviews with workshop coordinators and male and female counselors.

Key Words: Adolescent Sexuality, Sexual and Reproductive Health, Sexual Rights, Ethnic/Racial Differences, Colombia. 